\title{
Manifestações Orais da Leucemia no Momento do Diagnóstico
}

doi: https://doi.org/10.32635/2176-9745.RBC.2018v64n2.82

\author{
Oral Manifestations of Leukemia at the Time of Diagnosis \\ Manifestaciones Orales de Leucemia en el Momento del Diagnóstico
}

Michele Formighieri Antonini' ${ }^{\text {; }}$ Letícia Tainá de Oliveira Lemes²; Carolina Barreto Mozzini ${ }^{3}$

Resumo

Introdução: A leucemia é uma patologia com modificaçōes malignas nas células-tronco hematopoiéticas, podendo gerar sinais e sintomas no organismo do paciente. Objetivo: Verificar as manifestaçóes orais de leucemia no momento do diagnóstico. Método: Foi realizada uma revisão de literatura integrativa em maio e junho de 2018, a partir das bases de dados PubMed e BIREME, por meio das palavras cadastradas no MESH e no DeCS (Descritores em Saúde), respectivamente, em inglês leukemia AND diagnosis AND oral manifestations. Foram incluídos estudos avaliando as alteraçôes bucais em pacientes leucêmicos no momento do diagnóstico, publicados nas bases citadas, nas línguas portuguesa e inglesa, com qualquer desenho de estudo, exceto revisão de literatura narrativa e artigos publicados entre 1950 a 2018. Foram excluídos os artigos que não abordam o tema do estudo, que incluíram outros indivíduos, além de pacientes leucêmicos, com ausência de manifestaçôes orais em pacientes leucêmicos, e artigos não encontrados em sua versão completa. Resultados: Foram encontrados 353 artigos; destes, 318 foram excluídos. Assim, restaram 35 artigos. Conclusáo: Conforme descrito nos artigos encontrados, o cirurgião-dentista tem grande importância no momento do diagnóstico da leucemia, já que as primeiras manifestaçôes dessa doença ocorrem na cavidade oral, tais como sangramento gengival, hiperplasia, inchaço gengival, ulceração oral e petéquias.

Palavras-chave: Leucemia; Diagnóstico; Manifestações Bucais.

\begin{abstract}
Introduction: Leukemia is a pathology that consistent with modifications derived from hematopoietic stem cells, which can generate signs and symptoms in the patient's body. Objective: To verify the oral manifestations of leukemia at the time of diagnosis. Method: A review of the integrative literature was carried out in May and June of 2018 from the PubMed and BIREME databases, using the words registered in the MESH and the DeCS (Health Descriptors), respectively, in English leukemia AND diagnosis AND oral manifestations. We included studies evaluating oral alterations in leukemic patients at the time of diagnosis, published in the bases cited in Portuguese and English, with any study design, except revision of narrative literature and articles published between 1950 to 2018. We excluded articles that did not address the subject of the study, which included individuals other than leukemic patients, with absence of oral manifestations in leukemic patients and articles not found in its full version. Results: Through the search, 353 articles were found, of which 318 were excluded. Thus, 35 articles remained. Conclusion: According to the articles found, the surgeon-dentist has great importance at the time of diagnosis of leukemia, since the first manifestations of this disease occur in the oral cavity, such as gingival bleeding, hyperplasia, gingival swelling, oral ulceration and petechiae.

Key words: Leukemia; Diagnosis; Oral Manifestations.
\end{abstract}

\section{Resumen}

Introducción: La leucemia es una patología con modificaciones de las células madre hematopoyéticas, las cuales pueden generar signos y síntomas en el organismo del paciente. Objetivo: Verificar las manifestaciones orales de leucemia en el momento del diagnóstico. Método: Se realizó una revisión de literatura integrativa en mayo y junio de 2018 a partir de las bases de datos PubMed y BIREME, por medio de las palabras catastradas en el MESH y en el DeCS (Descriptores en Salud), respectivamente, en inglés leucemia AND diagnóstico AND oral manifestaciones y, Se incluyeron los estudios que evalúan las alteraciones orales en pacientes leucémicos el momento del diagnóstico, publicados en las bases mencionadas en portugués y en Inglés, con cualquier diseño de estudio, excepto la narrativa revisión de la literatura y artículos publicados entre 1950 e 2018. Se excluyeron los artículos que no abordan el tema del estudio, que incluyeron a otros individuos además de pacientes leucémicos, con ausencia de manifestaciones orales en pacientes leucémicos y artículos no encontrados en su versión completa. Resultados: A través de una búsqueda realizada encontraron 353 artículos, de éstas, 318 fueron excluídos. Así, quedaron 35 artículos. Conclusión: Según los artículos encontrados, el cirujano dentista tiene gran importancia en el momento del diagnóstico de la leucemia, ya que las primeras manifestaciones de esta enfermedad ocurren en la cavidad oral, tales como sangrado gingival, hiperplasia, hinchazón gingival, ulceración oral y petequias.

Palabras clave: Leucemia; Diagnóstico; Manifestaciones Bucales.

${ }^{1}$ Cirurgiã-Dentista. Faculdade Especializada na Área de Saúde. Passo Fundo (RS), Brasil. E- mail:micheleformighieri@gmail.com. Orcid ID: https://orcid.org/00000003-0612-6045

${ }^{2}$ Acadêmica do Programa de Pós-Graduação em Odontologia da Faculdade Meridional (IMED). Passo Fundo (RS), Brasil. E-mail: leticiatlemes@hotmail.com. Orcid ID: https://orcid.org/0000-0002-6525-5368

${ }^{3}$ Preceptora da Residência Multiprofissional em Saúde do Hospital da Cidade de Passo Fundo. Passo Fundo (RS), Brasil. E-mail: carolmozzini@gmail.com. Orcid ID: https://orcid.org/0000-0003-4360-2936

Endereço para correspondência: Polus Care Centro Clínico. Rua Bento Gonçalves, 900, sala 101, Centro. Passo Fundo (RS), Brasil. CEP $99020-060$. 


\section{INTRODUÇÃO}

A leucemia é um distúrbio hematológico, que se caracteriza pela diferenciação e proliferação de leucócitos malignos, que destroem a medula óssea, gerando a multiplicação de células leucêmicas ${ }^{1-7}$.

Segundo Silva et al. ${ }^{8}$, a leucemia representa um terço de todos os cânceres infantis, sendo que $75 \%$ dos pacientes pediátricos sofrem de leucemia linfoblástica aguda, que pode ser de origem das células $\mathrm{B}$ ou $\mathrm{T}$.

As causas dessa patologia são desconhecidas e incertas, muito embora se associe à exposição à radiação, anomalias cromossômicas, lesôes químicas e infecçôes virais, podendo ser causa da combinação de fatores ambientais e genéticos ${ }^{2,9}$.

As leucemias são classificadas de acordo com o seu curso clínico (agudo ou crônico) e, ainda, pela sua origem em histogenética (linfoide e mieloide). A leucemia crônica envolve relativamente os leucócitos e tem sua manifestação mais lenta, enquanto a leucemia aguda apresenta-se sob a forma mais rápida e gradativa ${ }^{2,5,10-12}$.

As alteraçôes estão associadas à diminuiçãa do número de leucócitos que, ao comprometer o sistema hematopoiético, gera sinais e sintomas no organismo do paciente como fadiga, cansaço e dispneia, além de alteraçôes na cavidade oral, apresentando aumento gengival, petéquias, equimose, sangramentos espontâneos, ulceração, líquen plano, hemorragias, hiperplasia, eritema multiforme, lúpus eritematoso, pênfigo vulgar e mucosite ${ }^{13-24}$.

Dessa forma, este estudo visa a investigar as manifestaçôes orais da leucemia no momento do diagnóstico e, assim, colaborar com o conhecimento da detecção de qualquer alteração existente na boca que indique outras patologias além das odontológicas. Ademais, visa a averiguar a idade que apresenta maior índice de acometimento na cavidade oral e, ainda, o papel do cirurgião-dentista no momento do diagnóstico.

\section{MÉTODO}

Este estudo trata de uma revisão de literatura integrativa sobre as manifestaçóes orais da leucemia no momento do diagnóstico. Foram consultados os bancos de dados eletrônicos: PubMed (MEDLINE) e Biblioteca Virtual em Saúde (BIREME). A pesquisa foi realizada em maio e junho de 2018, por meio da estratégia de busca que abordou o Mesh e entry terms no PubMed e palavraschave cadastradas no DeCS na BIREME.

A estratégia de busca foi a seguinte: no PubMed por meio do Mesh e entry terms - "Leukemia"[Mesh] OR "Leukemias" OR "Leucocythaemia" OR "Leucocythaemias" $O R$ "Leucocythemia" OR "Leucocythemias" AND "Oral Manifestations"[Mesh] OR "Manifestation, Oral" $O R$ "Manifestations, Oral" OR "Oral Manifestation" AND "Diagnosis"[Mesh] OR "Diagnoses" OR "Diagnoses and Examinations" $O R$ "Examinations and Diagnoses" $O R$ "Antemortem Diagnosis" OR "Antemortem Diagnoses" OR "Diagnoses, Antemortem" OR "Diagnosis, Antemortem". $\mathrm{Na}$ BIREME, por intermédio de palavras cadastradas no DeCS: "leukemia" AND "diagnosis" AND "oral manifestations". A busca foi ampliada para "leukemia" $A N D$ "diagnosis" AND "oral manifestation", sendo que a palavra "oral manifestation" não está cadastrada no DeCS.

O trabalho teve como critérios de inclusão: estudos referindo as alteraçôes bucais em pacientes leucêmicos no momento do diagnóstico; publicados nas bases citadas na língua portuguesa e inglesa; com qualquer desenho de estudo, exceto revisão de literatura narrativa; e publicados entre 1950 e 2018. Foram excluídos os artigos que não tratavam do tema do estudo; que incluíram outros indivíduos além de pacientes leucêmicos; aqueles que não continham as manifestaçôes orais em pacientes leucêmicos; e artigos que não foram encontrados em sua versão completa nem após solicitação para aquisição.

Os estudos foram analisados por dois pesquisadores independentes pelos seus títulos e resumos conforme os critérios de inclusão e exclusão. Após os artigos serem incluídos, passaram por uma análise de texto completo de acordo com os critérios de elegibilidade. Os dados extraídos foram relacionados às manifestaçóes orais, à idade dos pacientes e ao papel do cirurgiâo-dentista no diagnóstico da leucemia.

\section{RESULTADOS}

Por meio da busca realizada, foram encontrados 353 artigos, os quais foram selecionados mediante critérios de inclusão e exclusão do estudo. Dessa forma, foram incluídos 35 artigos. O detalhamento dos estudos encontra-se no Quadro 1. 
Quadro 1. Descrição dos relatos de caso elegíveis

\begin{tabular}{|c|c|c|c|c|}
\hline Referências & Formas de avaliação & Manifestações orais & $\begin{array}{l}\text { Idade dos } \\
\text { pacientes }\end{array}$ & $\begin{array}{c}\text { Condutas do cirurgião- } \\
\text {-dentista }\end{array}$ \\
\hline $\begin{array}{l}\text { Alessandrini } \\
\text { et al. (2012) }\end{array}$ & $\begin{array}{l}\text { Exames intraoral, } \\
\text { tomografia, } \\
\text { hemograma, análise } \\
\text { imuno-histoquímica, } \\
\text { biópsia e exame } \\
\text { extraoral }\end{array}$ & $\begin{array}{l}\text { Inchaço no vestíbulo superior } \\
\text { esquerdo, massa dolorosa } \\
\text { à palpação, firme e em } \\
\text { consistência elástica e lisa }\end{array}$ & 74 anos & --- \\
\hline $\begin{array}{l}\text { Anil et al. } \\
\text { (1996) }\end{array}$ & $\begin{array}{c}\text { Exames intra e } \\
\text { extraoral, de sangue e } \\
\text { biópsia }\end{array}$ & $\begin{array}{l}\text { Alargamento gengival e } \\
\text { dificuldade de mastigar }\end{array}$ & 34 anos & $\begin{array}{c}\text { Após realizar exames intra } \\
\text { e extraoral e solicitar exame } \\
\text { de sangue, cujo resultado } \\
\text { não apresentou alteração, } \\
\text { o cirurgião-dentista realizou } \\
\text { uma gengivectomia e mandou } \\
\text { amostra para biópsia, } \\
\text { suspeitando de leucemia }\end{array}$ \\
\hline $\begin{array}{l}\text { Appel e } \\
\text { Miggantz } \\
\text { (1988) }\end{array}$ & $\begin{array}{l}\text { Exames intraoral, } \\
\text { radiográfico, biópsia } \\
\text { da região da papila } \\
\text { entre os molares, } \\
\text { biópsia da medula } \\
\text { óssea e exames } \\
\text { laboratoriais }\end{array}$ & $\begin{array}{c}\text { Dor na região direita da } \\
\text { mandíbula, mucosa alveolar } \\
\text { vermelho--magenta, } \\
\text { eritema, papila e gengiva } \\
\text { com a aspecto purulento. } \\
\text { O molar inferior direito } \\
\text { demonstrou destruição } \\
\text { periodontal avançada e } \\
\text { cálculo subgengival. Perda } \\
\text { óssea generalizada horizontal, } \\
\text { cárie no terceiro molar inferior } \\
\text { direito, cálculo subgengival e } \\
\text { bolsa periodontal de } 8 \text { a } 10 \\
\text { mm }\end{array}$ & 59 anos & $\begin{array}{l}\text { O cirurgião-dentista realizou } \\
\text { o primeiro atendimento ao } \\
\text { paciente, o encaminhou para } \\
\text { um periodontista que solicitou } \\
\text { biópsia da papila bucal } \\
\text { entre os dentes inferiores do } \\
\text { lado esquerdo e primeiros } \\
\text { e segundos molares. Feito } \\
\text { isso, foi encaminhado ao } \\
\text { hospital para tratamento com } \\
\text { oncologista }\end{array}$ \\
\hline $\begin{array}{l}\text { Appel e } \\
\text { Miggantz } \\
\text { (2008) }\end{array}$ & $\begin{array}{l}\text { Hemograma completo, } \\
\text { exame clínico, } \\
\text { biópsia, exames } \\
\text { histopatológico e } \\
\text { intraoral }\end{array}$ & $\begin{array}{l}\text { Dor na região posterior } \\
\text { esquerda, dor e sangramento } \\
\text { gengival, edema no lado } \\
\text { esquerdo em torno do maxilar } \\
\text { inferior, trismo e eritema } \\
\text { localizado }\end{array}$ & 18 anos & -- \\
\hline $\begin{array}{l}\text { Babu et al. } \\
(2014)\end{array}$ & $\begin{array}{l}\text { Exames extra e } \\
\text { intraoral e hemograma } \\
\text { completo }\end{array}$ & $\begin{array}{l}\text { Sangramento e inchaço } \\
\text { gengival, linfadenopatia } \\
\text { cervical submandibular } \\
\text { profunda e bilateral; gengiva } \\
\text { eritematosa, placa e cálculo } \\
\text { dental, aumento generalizado } \\
\text { do maxilar e gengiva } \\
\text { mandibular envolvendo o } \\
\text { vestibulolingual }\end{array}$ & 43 anos & $\begin{array}{c}\text { O cirurgião-dentista, } \\
\text { após solicitar os exames } \\
\text { extraoral, sistêmico, intraoral } \\
\text { e hemograma completo, } \\
\text { instruiu o paciente a realizar } \\
\text { a escovação com escova de } \\
\text { dentes com cerdas macias } \\
\text { e a fazer bochechos de } \\
\text { clorexidina }(0,2 \%) 3 \text { vezes } \\
\text { ao dia, e o encaminhou } \\
\text { imediatamente para o centro } \\
\text { de gestão de oncologia }\end{array}$ \\
\hline $\begin{array}{l}\text { Boddu et al. } \\
(2017)\end{array}$ & $\begin{array}{c}\text { Exames intra e } \\
\text { extraoral, tomografia } \\
\text { computadorizada e } \\
\text { biópsia }\end{array}$ & $\begin{array}{l}\text { Dor gengival e lesões orais, } \\
\text { ulcerações sublinguais }\end{array}$ & 62 anos & -- \\
\hline $\begin{array}{l}\text { Brenneis, } \\
\text { Mattson e } \\
\text { Commers } \\
\text { (1988) }\end{array}$ & $\begin{array}{l}\text { Exames clínico e de } \\
\text { sangue }\end{array}$ & $\begin{array}{c}\text { Gengiva cianótica e } \\
\text { hiperplásica, linfadenopatia } \\
\text { cervical bilateral, e tecido } \\
\text { tonsilar alargado e } \\
\text { eritematoso }\end{array}$ & 43 anos & $\begin{array}{l}\text { O cirurgião-dentista solicitou } \\
\text { exame de sangue, pois o } \\
\text { paciente apresentava sinais } \\
\text { típicos de leucemia }\end{array}$ \\
\hline
\end{tabular}


Quadro 1. continuação

\begin{tabular}{|c|c|c|c|c|}
\hline Referências & Formas de avaliação & Manifestações orais & $\begin{array}{l}\text { Idade dos } \\
\text { pacientes }\end{array}$ & $\begin{array}{c}\text { Condutas do cirurgião- } \\
\text {-dentista }\end{array}$ \\
\hline $\begin{array}{l}\text { Bressman et } \\
\text { al. (1982) }\end{array}$ & $\begin{array}{l}\text { Exames clínico, } \\
\text { radiográfico, avaliação } \\
\text { hematológica, exame } \\
\text { físico e aspiração da } \\
\text { medula óssea }\end{array}$ & $\begin{array}{l}\text { Dor e sangramento } \\
\text { gengival no maxilar } \\
\text { direito na região do pré- } \\
\text { molar, gengiva marginal } \\
\text { fibrótica, dentes com } \\
\text { restaurações extensas e } \\
\text { perda óssea }\end{array}$ & 36 anos & $\begin{array}{l}\text { O paciente foi encaminhado } \\
\text { para o seu médico com um } \\
\text { pedido especial para uma } \\
\text { avaliação hematológica }\end{array}$ \\
\hline $\begin{array}{l}\text { Cale, } \\
\text { Freedman e } \\
\text { Lumerman } \\
\text { (1988) }\end{array}$ & Exames extra e intraoral & $\begin{array}{c}\text { Bolhas hemorrágicas no } \\
\text { dorso anterior da língua } \\
\text { e sangramento gengival } \\
\text { espontâneo }\end{array}$ & 45 anos & $\begin{array}{c}\text { Ao receber o paciente, o } \\
\text { cirurgião-dentista tratou o } \\
\text { paciente primeiramente com } \\
\text { vitamina C e amoxicilina, sem } \\
\text { solicitar qualquer exame ao } \\
\text { paciente }\end{array}$ \\
\hline $\begin{array}{l}\text { Chavan et } \\
\text { al. (2010) }\end{array}$ & $\begin{array}{c}\text { Exames clínicos intraoral e } \\
\text { de sangue }\end{array}$ & $\begin{array}{l}\text { Inchado doloroso e } \\
\text { sangramento gengival, } \\
\text { alteração da cor da } \\
\text { gengiva e papila } \\
\text { marginal, ulceração } \\
\text { gengival, equimose na } \\
\text { mucosa palatal dura e } \\
\text { mole }\end{array}$ & 35 anos & $\begin{array}{l}\text { O cirurgião-dentista solicitou } \\
\text { uma investigação hematológica } \\
\text { em razão da extensão e da } \\
\text { gravidade das alterações } \\
\text { gengivais. Após o diagnóstico, } \\
\text { foi encaminhado a um } \\
\text { oncologista }\end{array}$ \\
\hline $\begin{array}{l}\text { Chung et al. } \\
(2011)\end{array}$ & $\begin{array}{l}\text { Exames físico, } \\
\text { hemograma, intraoral e } \\
\text { radiográfico, tomografia } \\
\text { computadorizada e } \\
\text { biópsia }\end{array}$ & $\begin{array}{l}\text { Dor na região posterior } \\
\text { da mandíbula direita e } \\
\text { mobilidade nos dentes } \\
\text { anteriores inferiores }\end{array}$ & 35 anos & $\begin{array}{c}\text { O cirurgião-dentista realizou uma } \\
\text { anamnese, verificando o histórico } \\
\text { oral, e solicitou sua internação } \\
\text { para avaliação sistêmica do } \\
\text { paciente }\end{array}$ \\
\hline $\begin{array}{l}\text { Claus e } \\
\text { Denver } \\
(1954)\end{array}$ & $\begin{array}{l}\text { Exames laboratorial e } \\
\text { físico }\end{array}$ & -- & 37 anos & -- \\
\hline $\begin{array}{l}\text { Cooper, } \\
\text { Loewen e } \\
\text { Shore }(2000)\end{array}$ & $\begin{array}{c}\text { Exames intraoral e } \\
\text { radiográfico, de sangue e } \\
\text { biópsia }\end{array}$ & $\begin{array}{c}\text { Hiperplasia e } \\
\text { sangramento gengival }\end{array}$ & 35 anos & -- \\
\hline $\begin{array}{l}\text { Dean, } \\
\text { Ferguson } \\
\text { e Marvan } \\
\text { (2003) }\end{array}$ & $\begin{array}{c}\text { Exames extra e intraoral, } \\
\text { de sangue completo e } \\
\text { radiográfico }\end{array}$ & $\begin{array}{l}\text { Dor, ulceração gengival } \\
\text { e na mucosa, petéquias }\end{array}$ & 22 anos & $\begin{array}{c}\text { Medicou o paciente e o } \\
\text { encaminhou para um } \\
\text { especialista }\end{array}$ \\
\hline $\begin{array}{l}\text { Demirer et } \\
\text { al. (2007) }\end{array}$ & $\begin{array}{l}\text { Exames clínico, } \\
\text { radiográfico, hemograma } \\
\text { completo e biópsia da } \\
\text { medula óssea }\end{array}$ & $\begin{array}{c}\text { Hiperplasia gengival, } \\
\text { áreas eritematosas na } \\
\text { gengiva }\end{array}$ & 17 anos & -- \\
\hline $\begin{array}{l}\text { Fatahzadeh } \\
\text { e Krakow } \\
(2008)\end{array}$ & $\begin{array}{c}\text { Exames clínico, } \\
\text { radiográfico, intra e } \\
\text { extraoral, hemograma } \\
\text { completo, biópsia da } \\
\text { medula óssea e análise } \\
\text { do sangue periférico, } \\
\text { esfregaço }\end{array}$ & $\begin{array}{c}\text { Dor intermitente, } \\
\text { sangramento gengival } \\
\text { generalizado, gengiva } \\
\text { edemaciada e } \\
\text { eritematosa, placa, } \\
\text { tártaro, linfadenopatia } \\
\text { submandibular }\end{array}$ & 26 anos & -- \\
\hline $\begin{array}{l}\text { Guan e Firth } \\
(2015)\end{array}$ & $\begin{array}{c}\text { Exames extra e intraoral, } \\
\text { e de sangue }\end{array}$ & $\begin{array}{c}\text { Sangramento gengival } \\
\text { espontâneo }\end{array}$ & 49 anos & -- \\
\hline
\end{tabular}


Quadro 1. continuação

\begin{tabular}{|c|c|c|c|c|}
\hline Referências & Formas de avaliação & Manifestações orais & $\begin{array}{l}\text { Idade dos } \\
\text { pacientes }\end{array}$ & $\begin{array}{c}\text { Condutas do cirurgião- } \\
\text {-dentista }\end{array}$ \\
\hline $\begin{array}{l}\text { Gordon, } \\
\text { O'Neal e } \\
\text { Woodyard } \\
\text { (1985) }\end{array}$ & $\begin{array}{c}\text { Exames clínico, de } \\
\text { urina, hemograma } \\
\text { completo, soro glutâmico } \\
\text { oxalacética }\end{array}$ & $\begin{array}{l}\text { Palidez da mucosa oral, } \\
\text { gengiva firme, não } \\
\text { transcendente, com } \\
\text { contornos fisiológicas } \\
\text { normais }\end{array}$ & 42 anos & $\begin{array}{l}\text { Depois de o cirurgião- } \\
\text {-dentista tratar um quadro de } \\
\text { periodontite com raspagem e } \\
\text { alisamento radicular, o paciente } \\
\text { foi submetido a uma cirurgia } \\
\text { periodontal. Após um mês da } \\
\text { cirurgia, retornou com sintomas } \\
\text { de infecção respiratória, foi } \\
\text { medicado e solicitado exame de } \\
\text { sangue. Ao saírem os resultados, } \\
\text { o paciente foi encaminhado para } \\
\text { o hospital }\end{array}$ \\
\hline $\begin{array}{l}\text { Gowda et al. } \\
(2013)\end{array}$ & $\begin{array}{l}\text { Exames extra e intraoral, } \\
\text { e laboratorial }\end{array}$ & $\begin{array}{c}\text { Inchaço, dor e } \\
\text { sangramento gengival, } \\
\text { linfadenopatia } \\
\text { submandibular bilateral, } \\
\text { necrose localizada e } \\
\text { descamação envolvendo } \\
\text { a papila interdental } \\
\text { e alteração de cor da } \\
\text { gengiva marginal }\end{array}$ & 28 anos & -- \\
\hline $\begin{array}{l}\text { Katz e Peretz } \\
(2002)\end{array}$ & $\begin{array}{l}\text { Exames extra e intraoral, } \\
\text { raio-x panorâmico e } \\
\text { exame de sangue }\end{array}$ & $\begin{array}{l}\text { Trismo, sensibilidade à } \\
\text { palpação nos nódulos } \\
\text { submandibulares } \\
\text { bilateralmente }\end{array}$ & 6 anos & $\begin{array}{c}\text { Após a avaliação inicial do } \\
\text { paciente, solicitar exame de } \\
\text { sangue em razão da debilitação } \\
\text { do paciente }\end{array}$ \\
\hline $\begin{array}{l}\text { Keene, } \\
\text { Hussman } \\
\text { e Bruner } \\
(1972)\end{array}$ & $\begin{array}{l}\text { Exames radiográfico e } \\
\text { intraoral }\end{array}$ & $\begin{array}{l}\text { Hiperplasia gengival, } \\
\text { dor, eritema difuso, } \\
\text { perda óssea } \\
\text { generalizada, } \\
\text { sangramento gengival }\end{array}$ & 54 anos & $\begin{array}{l}\text { O cirurgião-dentista solicitou um } \\
\text { hemograma completo antes de } \\
\text { realizar o tratamento periodontal }\end{array}$ \\
\hline $\begin{array}{l}\text { Komeno et } \\
\text { al. (2015) }\end{array}$ & $\begin{array}{l}\text { Exames intra e extraoral, } \\
\text { e de sangue }\end{array}$ & $\begin{array}{c}\text { Candidíase oral e } \\
\text { esofágica }\end{array}$ & 47 anos & -- \\
\hline $\begin{array}{l}\text { Menezes e } \\
\text { Rao (2012) }\end{array}$ & $\begin{array}{c}\text { Exames intraoral, } \\
\text { laboratoriais, hemograma } \\
\text { completo e biópsia da } \\
\text { medula }\end{array}$ & $\begin{array}{c}\text { Dor, inchaço e } \\
\text { sangramento da } \\
\text { gengiva maxilar e } \\
\text { mandibular, gengiva } \\
\text { alargada e edemaciada, } \\
\text { necrose gengival nas } \\
\text { margens dos dentes }\end{array}$ & 34 anos & $\begin{array}{l}\text { Solicitou uma investigação de } \\
\text { sangue de rotina que revelou } \\
\text { alteração sugestiva de leucemia }\end{array}$ \\
\hline $\begin{array}{l}\text { Pogrel } \\
\text { (1978) }\end{array}$ & $\begin{array}{l}\text { Exames clínico, } \\
\text { radiográfico e biópsia }\end{array}$ & $\begin{array}{l}\text { Havia hipertrofia } \\
\text { acentuada na } \\
\text { mandíbula incisiva, } \\
\text { área hipertrofiada } \\
\text { apresentava cor } \\
\text { roxa, sangramento, } \\
\text { mobilidade dos incisivos } \\
\text { inferiores e lesão } \\
\text { gengival }\end{array}$ & 32 anos & $\begin{array}{l}\text { O cirurgião-dentista realizou } \\
\text { uma biópsia na região de } \\
\text { hipertrofia da mandíbula dos } \\
\text { incisivos }\end{array}$ \\
\hline $\begin{array}{l}\text { Ratre et al. } \\
(2018)\end{array}$ & $\begin{array}{l}\text { Exames intra e extraoral, } \\
\text { e de sangue }\end{array}$ & $\begin{array}{c}\text { Gengiva inchada e } \\
\text { dificuldade para comer }\end{array}$ & 51 anos & -- \\
\hline $\begin{array}{l}\text { Reenesh, } \\
\text { Munishwar e } \\
\text { Rath (2012) }\end{array}$ & $\begin{array}{l}\text { Exames físico, intraoral, e } \\
\text { de sangue }\end{array}$ & $\begin{array}{l}\text { Dor e sangramento } \\
\text { gengival, gengiva com } \\
\text { cor azulada e com } \\
\text { presença de equimoses }\end{array}$ & 32 anos & -- \\
\hline
\end{tabular}


Quadro 1. continuação

\begin{tabular}{|c|c|c|c|c|}
\hline Referências & Formas de avaliação & Manifestações orais & $\begin{array}{l}\text { Idade dos } \\
\text { pacientes }\end{array}$ & $\begin{array}{c}\text { Condutas do cirurgião- } \\
\text {-dentista }\end{array}$ \\
\hline $\begin{array}{l}\text { Shimizu et } \\
\text { al. (2017) }\end{array}$ & $\begin{array}{c}\text { Exames físico, intraoral, } \\
\text { de sangue e tomografia } \\
\text { computadoriza }\end{array}$ & $\begin{array}{c}\text { Edema grave na região } \\
\text { vestibular direita, } \\
\text { aumentada no seio maxilar } \\
\text { direito por meio do exame } \\
\text { tomográfico }\end{array}$ & 12 anos & \\
\hline $\begin{array}{l}\text { Sepúlveda et } \\
\text { al. (2012) }\end{array}$ & $\begin{array}{l}\text { Exames intraoral e } \\
\text { hematológico }\end{array}$ & $\begin{array}{c}\text { Hiperplasia e } \\
\text { sangramento gengival, } \\
\text { petéquias e equimose, } \\
\text { linfadenopatias }\end{array}$ & 6 anos & -- \\
\hline $\begin{array}{l}\text { Sharma } \\
\text { e Bhalla } \\
(2011)\end{array}$ & $\begin{array}{c}\text { Exames intra e extraoral, } \\
\text { e de sangue }\end{array}$ & $\begin{array}{l}\text { Lesões herpetiforme } \\
\text { dolorosas na região } \\
\text { extraoral adjacente, } \\
\text { vermelhão do lábio } \\
\text { inferior, ulcerações } \\
\text { cobertas com manchas } \\
\text { esbranquiçadas. Foi } \\
\text { observada perda de } \\
\text { contornos normais e } \\
\text { ponteado da gengiva }\end{array}$ & 16 anos & -- \\
\hline $\begin{array}{l}\text { Shen et al. } \\
(2018)\end{array}$ & Exames intra e extraoral & $\begin{array}{c}\text { Inchaço gengival, } \\
\text { periodontite crônica e } \\
\text { epúlide }\end{array}$ & 41 & -- \\
\hline $\begin{array}{l}\text { Silva et al. } \\
(2008)\end{array}$ & $\begin{array}{l}\text { Exame físico, biópsia } \\
\text { da úlcera genital, } \\
\text { exames subsidiários, } \\
\text { hemograma, } \\
\text { citogenética e } \\
\text { imunofenotipagem }\end{array}$ & $\begin{array}{l}\text { Úlceras orais múltiplas } \\
\text { recorrentes com } \\
\text { dificuldade de cicatrização } \\
\text { e gengivite }\end{array}$ & $\begin{array}{l}10 \text { anos e } \\
\text { seis meses }\end{array}$ & -- \\
\hline $\begin{array}{l}\text { Silva et al. } \\
(2012)\end{array}$ & $\begin{array}{l}\text { Anamnese, exames } \\
\text { extra e intraoral, } \\
\text { mielograma, biópsia } \\
\text { de medula óssea e } \\
\text { imunofenotipagem }\end{array}$ & $\begin{array}{l}\text { Inchaço com consistência } \\
\text { fibrosa indolor à palpação }\end{array}$ & 10 anos & $\begin{array}{c}\text { O cirurgião-dentista } \\
\text { encaminhou o paciente para o } \\
\text { Departamento de Odontologia } \\
\text { do Hospital de Mato Grosso } \\
\text { para acelerar a avaliação e } \\
\text { consequentemente o tratamento }\end{array}$ \\
\hline $\begin{array}{l}\text { Stoopler et } \\
\text { al. (2004) }\end{array}$ & $\begin{array}{c}\text { Exames intra e } \\
\text { extraoral, tomografia } \\
\text { computadorizada, testes } \\
\text { hematológicos, biópsia } \\
\text { da medula e análise de } \\
\text { citometria }\end{array}$ & $\begin{array}{l}\text { Ulcerações, eritema oral, } \\
\text { úlceras neutropênicas, } \\
\text { herpes intraoral crônica e } \\
\text { infecção fúngica }\end{array}$ & 50 anos & -- \\
\hline $\begin{array}{l}\text { Tag-Adeen } \\
\text { et al. (2018) }\end{array}$ & $\begin{array}{l}\text { Exame clínico e } \\
\text { laboratoriais }\end{array}$ & Úlceras dolorosas na boca & 49 anos & -- \\
\hline $\begin{array}{l}\text { Wu, Fantasia } \\
\text { e Kaplan } \\
(2002)\end{array}$ & $\begin{array}{c}\text { Exame de sangue e } \\
\text { biópsia }\end{array}$ & $\begin{array}{l}\text { Inchaço gengival, } \\
\text { alargamento da gengiva } \\
\text { maxilar e mandibular } \\
\text { envolvendo o assoalho } \\
\text { bucal, lingual e aspectos } \\
\text { palatais; gengiva pálida, } \\
\text { bulbosa e petéquias } \\
\text { palatinas também } \\
\text { foram observadas, além } \\
\text { de nódulos cutâneos } \\
\text { envolvendo a face e a } \\
\text { testa }\end{array}$ & 53 anos & -- \\
\hline
\end{tabular}

Nota: -- Estudo não apresentou este dado. 


\section{DISCUSSÃO}

As manifestaçôes clínicas dos pacientes com leucemia crônica estão relacionadas com a diminuição da produção das células sanguíneas que podem acarretar em quadros de anemia, neutropenia, trombocitopenia ${ }^{4,17}$.

As alteraçōes orais com maior ocorrência na literatura em casos de leucemia confirmam-se nos resultados deste trabalho e são relatadas em diferentes estudos, tais como sangramento gengival ${ }^{3,16,18-24}$; inchaço gengiva $^{3,11,23,25,26}$; úlceras orais ${ }^{2,5,13,15,25,27-30}$; petéquias 2,5,27,28,31,32; hiperplasia, $5,10,21,27,29,31,33,34$.

Além dessas manifestaçóes, foi verificado relato de dor na regiáo maxilar e/ou mandibular ${ }^{2,3,18,20,32,33}$.

Outras manifestações também foram encontradas, as quais incluem mobilidade nos dentes anteriores inferiores ${ }^{9}$; linfadenopatia ${ }^{1,23,31,32}$; sensibilidade à palpação nos nódulos submandibulares bilateralmente ${ }^{7}$.

Aronovich e Connolly ${ }^{13}$ revelam uma condição inflamatória que ocorre em dentes impactados ou parcialmente erupcionados, conhecida por pericoronarite. Normalmente, essa manifestação acontece em terceiros molares no momento de sua erupção, onde a placa bacteriana e restos de comida se aderem debaixo da gengiva, proporcionando um substrato e meio para a infecção.

Sepúlveda et al. ${ }^{23}$ relatam que a leucemia é uma neoplasia cuja maior frequência ocorre em crianças menores de 15 anos. Por outro lado, Brenneise et al. ${ }^{24}$ relatam que a leucemia linfocítica aguda possui maior prevalência em jovens de 15 anos de idade. Enquanto a leucemia linfocítica crônica predomina em pacientes com 60 anos de idade.

As lesôes orais são muitas vezes o primeiro sinal ou sintoma de um quadro leucêmico, fazendo com que os pacientes procurem atendimento odontológico, acreditando que seja um problema de origem local e não sistêmico. Nesse sentido, o cirurgião-dentista é fundamental na avaliaçáo inicial do paciente, auxiliando assim no diagnóstico e no prognóstico da doença ${ }^{2,8}$. Educar os pacientes, enfatizando a prevenção e oferecendo modificaçôes nas rotinas de higiene bucal são algumas atitudes que podem ser tomadas ${ }^{10,13,22}$.

A avaliação do cirurgiáo-dentista consiste basicamente na realização dos exames intraoral e extraoral. Sendo que, para isso, poderá fazer uso de diferentes ferramentas para auxiliá-lo, tais como biópsia de áreas sugestivas à alteração ou da medula óssea.

Os exames intraoral e extraoral são relatados na maior parte dos artigos analisados, enquanto a biópsia, uma alternativa de diagnóstico simples, segura e menos invasiva, foi relatada por poucos estudos ${ }^{1,2,19,20,23,25,31,33}$. Porém, os artigos não demonstram seguir um parâmetro para a forma de avaliação. Não há critérios, nem especificação de como é feita a avaliação física, extraoral ou intraoral, nos casos relatados.

Em relação aos exames de imagem, as radiografias periapical e panorâmica não revelaram nenhuma anormalidade ${ }^{2,10,22}$. Em contrapartida, outros autores evidenciaram, no exame radiográfico, uma perda óssea ${ }^{20,23,27,33,34}$.

Nesse sentido, além de realizar um bom atendimento inicial do paciente, o cirurgiáo-dentista deve estar atento nâo só as manifestações, mas também no acompanhamento do paciente, tendo ciência das diferentes doenças sistêmicas e patologias passageiras.

As condutas odontológicas invasivas, a serem realizadas com esses pacientes, devem ser executadas com cautela em razão do alto risco de hemorragia e infecção nesses pacientes.

Observa-se uma grande dificuldade em relação aos desenhos de estudos encontrados, uma vez que a maioria das pesquisas nesse tema desenvolveu relatos de casos, e nenhum estudo observacional incluiu um maior número de pacientes. Nesse sentido, se alerta sobre a precariedade de informaçôes consistentes. Além disso, questionam-se as constantes publicaçôes envolvendo relatos sobre a mesma doença e abordadas de forma semelhantes.

\section{CONCLUSÃO}

Diante do exposto, a leucemia apresenta suas primeiras manifestaçóes na cavidade oral, as alteraçóes mais frequentes são sangramento gengival, hiperplasia, inchaço gengival, ulceração oral e petéquias. A idade com maior incidência de casos de leucemia e ocorrência de acometimentos orais foi entre 32 a 37 anos de idade. $\mathrm{O}$ cirurgião-dentista tem papel fundamental no diagnóstico rápido e precoce da doença, pois os pacientes com alteração na cavidade oral procuram inicialmente um dentista. Dessa forma, os profissionais possuem o primeiro contato com os sinais e sintomas dessa patologia, podendo solicitar exames que confirmem a suspeita de uma possível alteração, possibilitando assim um diagnóstico rápido e precoce da doença e proporcionando um melhor prognóstico aos pacientes. $\mathrm{Na}$ equipe multidisciplinar de oncologia, o dentista contribui com a equipe médica antes, durante e após o tratamento médico.

\section{CONTRIBUIÇÕES}

Todos os autores contribuíram igualmente em todas as etapas do estudo. 


\section{DECLARAÇÃO DE CONFLITO DE INTERESSES}

Nada a Declarar.

\section{REFERÊNCIAS}

1. Babu K, Kashyap VP, Sivaranjani P, Agila S. An undiagnosed case of acute myeloid Leukemia. Journal of Indian Society of Periodontology. 2014;18(1):95-97.

2. Dean AK, Ferguson JW, Marvan ES. Acute leukaemia presenting as oral ulceration to a dental emergency servisse. Australian Dental Journal. 2003;48(3):195-197.

3. Menezes L, Rao JR. Acute myelomonocytic leukemia presenting with gingival enlargement as the only clinical manifestation. Journal of Indian Society of Periodontology. 2012;16(4):597-601.

4. Reenesh M, Munishwar S, Rath SK. Generalised Leukaemic Gingival Enlargement: a Case Report. J Oral Maxillofac Res. 2012,3(3):e5.

5. Shen Y, Zhao L, Yafei WUY, HUANG P. Multifocal occurrence of intraoral isolated MS in a patient without leukemic presentation: A case report and literature review. Oral Medicine, New York, 2018;125(3):42-48.

6. Silva BA, Siqueira CRB, Castro PHS, Araujo SS, Volpato LER. Oral manifestations leading to the diagnosis of acute lymphoblastic leukemia in a young girl. Journal of Indian Society of Pedodontics and Preventive Sentistry. 2012;30(2):166-8.

7. Chung W, KIM S, CHOI JR. Osteolytic mandible presenting as an initial manifestation of an adult acute lymphoblastic leukaemia. Int. J. Oral Maxillofac. Surg. 2011;40:1438-1440.

8. Demirer S, Özdemir H, Mehmet Ş, Marakoğlu I. Gingival Hyperplasia as an Early Diagnostic Oral Manifestation in Acute Monocytic Leukemia: A Case Report. European Journal of Dentistry. 2007;1(2):111-114.

9. Silva K, Higa M, Terreri MTSLRA, Borsato ML, Hilário MOE. Ulceraçôes orais e genitais como manifestaçâoo inicial de leucemia em criança. Rev Paul Pediatr. 2008;26(1):84-87.

10. Ratre MS, Gulati R, Khetarpal S, Parihar A. Regular oral screening and vigilance: can it be a potential lifesaver?. J Indian Soc Periodontol, 2018;22(3):171-173.

11. Aronovich S, Connolly TW. Pericoronitis as an Initial Manifestation of Acute Lymphoblastic Leukemia: A Case Report. J Oral Maxillofac Surg. 2008;66(4):804-808.

12. Fatahzadeh M, Krakow AM. Manifestation of acute monocytic leukemia in the oral cavity: a case report. Spec Care Dentist. 2008;28(5):190-194.

13. Boddu P, Chenb PL, Nagarajan P, Priet VG, Won A, Chambers $\mathrm{M}$, et al. Necrotizing fungal gingivitis in a patient with acute myelogenous leukemia: Visible yet obscure. Journal of Oral and Maxillofacial Surgery, 2017;30(1):50-54.

14. Alessandrini M, Micarelli A, Mugnaini F, De-Padova A, Pavone I, Bruno E. Unusual case of oral chronic lymphocytic leukemia presenting as recurrent epistaxis and asymptomatic intraoral swelling. Rev Stomatol Chir Maxillofac. 2012;113:455-457.

15. Cale AE, Freedman PD, Lumerman H. Acute promylocytic leukemia appearing as spontaneous oral hemorrhage: report of case. JADA. 1988;116(6):671-672.

16. Bressman E, Decter JA, Chasens AI. Acute myeloblastic leukemia with oral Manifestations. Oral Surg. 1982;54(4):401-403.

17. Cooper CL, Loewen R, Shore T. Gingival hiperplasia complicating acute myelomonocytic leukemia. J Can Den Assoc. 2000;66(2):78-79.

18. Gowda TM, Thomas R, Shanmukhappa SM, Agarwal G, Mehta D. Gingival enlargement as an early diagnostic indicator in therapyrelated acute myeloid leukemia: A rare case report and review of literature. Journal of Indian Society of Periodontology, 2013;17(2):248-252.

19. Pogrel MA. Acute leukemia an atypical case presenting with gingival manifestations. Int. J. Oral Surg. 1978;7(4):119-122.

20. Chavan M, Subramaniam A, Jhaveri H, Khedkar S, Durkar SD, Argwal A. Acute myeloid leukemia: a case report with palatal and lingual gingival alterations. Braz J Oral Sci. 2010;9(1):67-69.

21. Wu J, Fantasia JE, Kaplan R. Oral manifestations of acute Myelomonocytic Leukemia: A Case Report and Review of the Classification of Leukemias. J. Periodontol. 2002;73(6):664-668.

22. Stoopler ET, Pinto A, Alawi F, Raghavendra S, BoycrJunior R, Porter D, et al. Granulocytic sarcoma: an atypical presentation in the oral cavity. Spec Care Dentist. 2004;24(2)65-69.

23. Sepúlveda E, Brethauer U, Fernández E, Cortés G, Mardones C. Oral Manifestations as First Clinical Sign of Acute Myeloid Leukemia: Report of a Case. Pediatric Dentistry. 2012;34(5):418-21.

24. Brenneise CV, Mattson JS, Commers JR. Acute myelomonocytic leukemia with oral manifestations: report of case. Jada. 1988;117(7):835-837.

25. Keene JJ, Hussman L, Bruner G. Terminal Oral Manifestations of Acute Lymphoblastic Leukemia. 1972;27(4):117-119.

26. Appel BN, Miggantz R J. Acute Nonlymphocytic Leukemia, Monocytic Variant Report of a Case. J. Periodontol, 1988;59(7):464-468.

27. Anil S, Smaranayake LP, Nair RG, Beena VT. Gingival enlargement as a diagnostic indicator in leukemia. Case report. Australian Dental Journal. 1996;41(4):235-237. 
28. Claus EC, Denver C. Oral Manifestations in a case of myelogenous leukemia and leukopenia. Oral Surg Oral Med Oral Pathol. 1954;7(6):616-619.

29. Sharma U, Bhalla S. Oral Manifestations of a Systemic Disease. J Can Dent Assoc. v. 2011;77:b71.

30. Komeno Y, Uryu H, Iwata Y, Hatada Y, Sakamoto J, Iihara $\mathrm{K}$, et al. Esophageal Candidiasis as the Initial Manifestation of Acute Myeloid Leukemia. Intern Med. 2015;54(7):3087-3092.

31. Guan G, Firth N. Oral manifestations as an early clinical sign of acute myeloid leukaemia: a case report. Send to Aust Dent J. 2015;60(1):123-7.

32. Katz J, Peretz B. Trismus in a 6 year old child: a manifestation of leukemia? J Clin Pediatr Dent. 2002;26(4):337-339.

33. Tag-Adeen M, Hashiguchi K, Akazawa Y, Ohnita K, Yasushi S, Daisuke N. et al. An unusual presentation of adult T-cell leukemia/lymphoma. Ecancermedicalscience. 2018;12:801.

34. Shimizu R, Ohga N, Miyakoshi M, Asaka T, Sato J, Kitagawa Y. Unusual maxillary osteoblastic and osteolytic lesions presenting as an initial manifestation of childhood acute myeloid leukemia: A case report. Quintessence Int. 2017;48(2):149-153.

35. Gordon MR, O'NEAL RB, WOODYARD SGA. Variation from classic oral manifestations associated with acute myeloblastic leukemia. A case report. J Periodontol. 1985;56(5):285-7. 